\title{
Relação entre a circunferência da árvore e a comunidade de formigas (Hymenoptera: Formicidae) arborícolas em uma área de preservação amazônica \\ Relationship between tree circumference and arboreal ants community (Hymenoptera: Formicidae) in a protected area in the Amazon
}

\author{
Joudellys Andrade-Silva ${ }^{\mathrm{l}, \mathrm{ll}}$ (D) | Rony Peterson Santos Almeida ${ }^{\mathrm{l}, \mathrm{ll}}$ (1) \\ IMuseu Paraense Emílio Goeldi/MCTIC. Belém, Pará, Brasil \\ "Universidade Federal do Pará. Programa de Pós-Graduação em Zoologia. Belém, Pará, Brasil
}

\begin{abstract}
Resumo: Mecanismos responsáveis pela coexistência de espécies no ambiente têm implicações consideráveis na estruturação da fauna local. Aqui, avaliamos a estrutura da comunidade de formigas em uma área de floresta amazônica preservada, utilizando a circunferência das árvores como um parâmetro de heterogeneidade ambiental. Para isso, selecionamos 29 árvores, com diferentes circunferências a altura do peito (de 11 a $234 \mathrm{~cm}$ ), e capturamos as formigas que utilizam esse substrato através de pitfalls com urina humana. Encontramos 30 espécies de formigas, mas riqueza e composição não foram influenciadas pela circunferência das árvores. Fatores combinados e mais diretamente associados à heterogeneidade ambiental, como a conectividade das árvores, devem ser levados a efeito, a fim de que tenhamos conhecimento sobre os impulsionadores locais de diversidade no estrato vertical de remanescentes amazônicos preservados.
\end{abstract}

Palavras-chave: Ambientes preservados. Floresta Nacional de Caxiuanã. Estrato vertical. Amazônia brasileira. Formigas neotropicais.

Abstract: The mechanisms responsible for the coexistence of species in the environment have considerable implications in the structuring of local fauna. We aim to evaluate the structure of the ant community in a protected area in the Amazon rainforest, using trees circumference as a parameter of environmental heterogeneity. We conducted this experiment on 29 trees from different circumferences (from $11 \mathrm{~cm}$ to $234 \mathrm{~cm}$ ), and captured the ants using pitfall traps with human urine. We found 30 ant species, but the tree circumference did not influence either the richness or the composition of ants. Studies on combined factors and more directly associated with environmental heterogeneity, such as the connectivity of trees, must be carried out so that we can understand the local drivers of diversity in the vertical stratum of preserved Amazon forest remnants.

Keywords: Preserved environments. Caxiuanã National Forest. Vertical stratum. Brazilian Amazon. Neotropical ants.

ANDRADE-SILVA, J. \& R. P. S. ALMEIDA, 2020. Relação entre a circunferência da árvore e a comunidade de formigas (Hymenoptera: Formicidae) arborícolas em uma área de preservação amazônica. Boletim do Museu Paraense Emílio Goeldi. Ciências Naturais 15(1): 145-153. DOI: http://doi.org/10.46357/bcnaturais.v15i1.287.

Autor para correspondência: Joudellys Andrade-Silva. Museu Paraense Emílio Goeldi/MCTIC. Coordenação de Ciências da Terra e Ecologia. Av. Perimetral, 1901 - Terra Firme. Belém, PA, Brasil. CEP 66077-830 (joudellys@gmail.com).

Recebido em 26/02/2020

Aprovado em 06/04/2020

Responsabilidade editorial: Lívia Pires do Prado
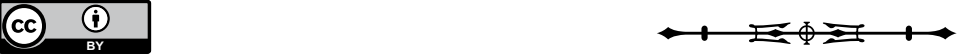


\section{INTRODUÇÃO}

Diferentes processos atuam na determinação e regulação das comunidades ecológicas (Ricklefs \& Schluter, 1993). Em habitats estruturalmente complexos, a riqueza de espécies de formigas (Hymenoptera: Formicidae) é positivamente associada à estrutura do habitat, principalmente em locais com alta disponibilidade de recursos e de locais para forrageamento e nidificação (Klimes et al., 2012), como em florestas tropicais.

A disponibilidade de recursos e de sítios para nidificação é componente importante na estruturação das comunidades de formigas (Fagundes et al., 2015). Para formigas arborícolas tropicais, este recurso é fornecido pelas árvores (Ribas et al., 2003). Portanto, o forrageamento, ou mesmo a colonização nesse substrato, depende do nível de qualidade e disponibilidade de alimento, fatores ainda mais relevantes para formigas que utilizam o estrato arbóreo como local para seu desenvolvimento biológico (Tanaka et al., 2010).

Comunidades de formigas arborícolas são estruturadas por diferentes fatores, como especificidade da planta hospedeira (Yamazaki et al., 2016), estágio sucessional (Sousa-Souto et al., 2016), além do espaço e da estratégia de forrageamento adotado pela(s) espécie(s). Dolichoderus Lund, 1831, por exemplo, possui maior atividade durante o dia, enquanto Acanthoponera Mayr, 1882 apresenta hábitos predominantemente noturnos (Baccaro et al., 2015). Além disso, muitas outras formigas arborícolas forrageiam no solo principalmente durante a noite, quando a riqueza de espécies de formigas epigeicas é menor (Hashimoto et al., 2010). Ainda, há espécies que se especializaram em forragear ou nidificar no solo e em outras partes da vegetação (Campos et al., 2008), o que impulsiona a diversidade e possibilita a coexistência interespecíica.

Portanto, nosso objetivo foi avaliar a estrutura da comunidade de formigas em uma área de floresta amazônica preservada, buscando entender se existe relação entre a organização da comunidade de formigas (riqueza e composição de espécies) e a circunferência das árvores que elas ocupam. Para isso, hipotetizamos que árvores com maiores circunferências (i) comportam maior riqueza de formigas, uma vez que oferecem maiores áreas para forrageamento e sítios para nidificação; e (ii) impulsionam mudanças na composição específica de formigas, haja vista a associação desta característica com mecanismos que promovem a coexistência de espécies.

\section{MATERIAL E MÉTODOS}

\section{ÁREA DE ESTUDO}

Realizamos o estudo em uma área de terra firme localizada na Estação Científica Ferreira Penna (ECFPn; $1^{\circ} 42^{\prime} 30^{\prime \prime}$ S; 51 31' 45" W; Figura 1), um sítio de pesquisa existente na Floresta Nacional (FLONA) de Caxiuanã, na região norte do estado do Pará, Brasil. A FLONA Caxiuanã é uma área primária de floresta amazônica com 330.000 ha, que apresenta, além da floresta de terra firme, várzeas e igapó, manchas de savana e vegetação secundária (Lisboa, 1997). A vegetação é densa, de dossel fechado e com árvores que podem alcançar $50 \mathrm{~m}$ de altura (Silva et al., 2003).

\section{DESENHO AMOSTRAL}

Realizamos as coletas nos dias 28 e 29 de novembro de 2017, período que corresponde à estação seca na região. Ao longo de um transecto de 600 m e com auxílio de uma fita métrica, selecionamos 29 árvores, distantes pelo menos $20 \mathrm{~m}$ entre si. Direcionamos a escolha das árvores de modo a preencher um gradiente de circunferência à altura do peito (CAP), variando entre $10 \mathrm{~cm}$ e $234 \mathrm{~cm}$. De forma alternada, selecionamos uma árvore entre ambos os lados (direito e esquerdo) ao longo do transecto. Estabelecemos a distância mínima entre árvores a partir da última árvore marcada, independentemente do lado amostrado.

\section{COLETA E IDENTIFICAÇÃO DAS FORMIGAS}

Em cada árvore selecionada, inserimos dois pitfalls feitos com copos plásticos (180 mL; para detalhes, ver 


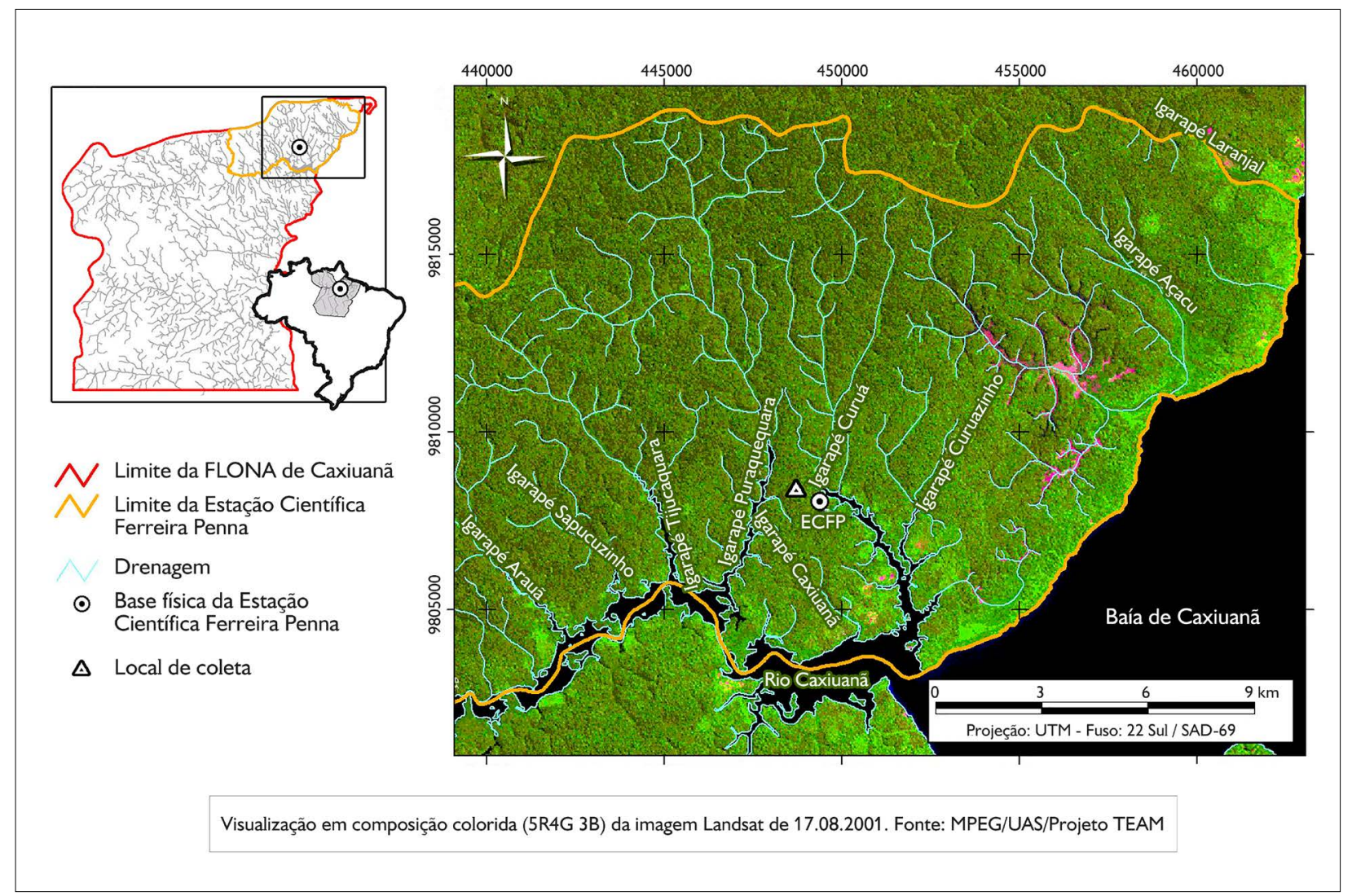

Figura 1. Área de amostragem de formigas na Floresta Nacional de Caxiuanã, Melgaço, Pará, Brasil. Mapa: MPEG/UAS/Projeto TEAM.

Bestelmeyer et al., 2000) - um em cada lado, fixados ao tronco a 2 m de altura. Em cada copo, adicionamos uma solução de urina humana diluída em água, na proporção de 1:3, e acrescida de gotas de detergente neutro. Armadilha utilizando urina como atrativo vem se mostrando eficiente em campo e tem sido utilizada nas abordagens mais recentes sobre formigas em estrato vertical (Powell et al., 2011; Vasconcelos et al., 2017).

Os pitfalls permaneceram expostos no tronco de cada árvore por 24 horas. Ao final da exposição, retiramos as formigas da solução e armazenamos em tubos eppendorfs contendo álcool 96\%. No laboratório da ECFPn, processamos e montamos todo o material coletado. Fizemos as identificações das formigas até o nível de gênero, seguindo a chave de identificação fornecida por Baccaro et al. (2015); realizamos as identificações em nível de espécie por meio de comparações com a Coleção Entomológica do Museu Paraense Emílio Goeldi, onde depositamos a coleção de referência.

\section{ANÁLISES DOS DADOS}

Para avaliarmos se árvores com maiores circunferências à altura do peito (CAP) comportam maior riqueza de espécies de formigas, construímos um modelo linear generalizado (GLM) com distribuição quasipoisson, aplicando o teste F de Fisher (Crawley, 2013). Para esta análise, utilizamos a função "glm" do pacote stats (R Core Team, 2019). A validação do modelo seguiu o protocolo gráfico de Zuur et al. (2010).

Para avaliarmos se maiores CAP impulsionam mudanças na composição de espécies de formigas, utilizamos uma análise multivariada de permutação

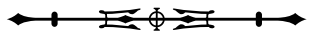


(PERMANOVA) com 9.999 aleatorizações (Clarke, 1993), e considerando a similaridade de Jaccard. Para esta análise, utilizamos a função "adonis" do pacote vegan (Oksanen et al., 2019).

Para a visualização gráfica, utilizamos os pacotes ggplot2 (Wickham, 2016) e gridExtra (Auguie, 2017). Realizamos todas as análises no software R (R Core Team, 2019) com nível de significância de 5\%.

\section{RESULTADOS}

Coletamos 30 espécies de formigas, divididas em 12 gêneros e seis subfamílias (Tabela 1). Dolichoderus Lund, 1831 (seis espécies), Camponotus Mayr, 1861 (cinco) e Crematogaster Lund, 1831 (quatro) foram os gêneros de maiores riquezas. As espécies com maiores frequências de ocorrências nas árvores foram Crematogaster levior Longino, 2003 (11 árvores), Camponotus femoratus (Fabricius, 1804) (10) e Camponotus atriceps (Smith, 1858) (cinco).

Nossa hipótese de que árvores com maiores circunferências comportam maior riqueza de formigas não foi corroborada $\left(F_{(1,27)}=3,525 ; p=0,071\right.$; Figura 2). Ainda, a hipótese de que maiores circunferências arbóreas atuam como impulsionadoras de mudanças na composição específica de formigas também foi refutada (Pseudo $\mathrm{F}_{(1,26)}$ $=1,481 ; R^{2}=0,054 ; p=0,102 ;$ Figura 3).

\section{DISCUSSÃO}

Neste estudo, utilizamos um gradiente de circunferência à altura do peito (CAP) para verificar a sua relação com a estrutura da comunidade de formigas arbóreas em um fragmento preservado de floresta amazônica. Nossos resultados indicam que este parâmetro da comunidade vegetal não explica as alterações na riqueza e composição de formigas, refutando nossa hipótese de que a CAP poderia ser uma importante moduladora da diversidade local de formigas arbóreas. Esse resultado pode estar associado a diversos fatores relacionados à complexidade estrutural da vegetação (Stein et al., 2014) ou mesmo ao comportamento, em muitos casos agressivos e
Tabela 1. Ocorrência das espécies de formigas em 29 árvores amostradas na Floresta Nacional de Caxiuanã, Melgaço, Pará, Brasil.

\begin{tabular}{|c|c|}
\hline Subfamília/Espécie & $\begin{array}{c}\text { Número de } \\
\text { árvores }\end{array}$ \\
\hline \multicolumn{2}{|l|}{ Dolichoderinae } \\
\hline Azteca sp.1 & 4 \\
\hline Azteca sp.2 & 1 \\
\hline Dolichoderus attelaboides (Fabricius, 1775) & 1 \\
\hline Dolichoderus bidens (Linnaeus, 1758) & 2 \\
\hline Dolichoderus bispinosus (Olivier, 1792) & 1 \\
\hline Dolichoderus decollatus Smith, 1858 & 1 \\
\hline Dolichoderus imitator Emery, 1894 & 1 \\
\hline Dolichoderus aff. inpai & 1 \\
\hline \multicolumn{2}{|l|}{ Ectatomminae } \\
\hline Ectatomma tuberculatum (Olivier, 1792) & 1 \\
\hline Gnamptogenys sp.1 & 1 \\
\hline Gnamptogenys sp.2 & 1 \\
\hline
\end{tabular}

\begin{tabular}{c|c}
\hline \multicolumn{1}{l}{ Formicinae } \\
\hline Camponotus apicalis (Mann, 1916) & 1 \\
\hline Camponotus atriceps (Smith, 1858) & 5 \\
\hline Camponotus femoratus (Fabricius, 1804) & 10 \\
\hline Camponotus sp.2 & 1 \\
\hline Camponotus sp.5 & 2 \\
\hline
\end{tabular}

\begin{tabular}{c|c}
\hline \multicolumn{1}{l}{ Myrmicinae } \\
\hline Cephalotes atratus (Linnaeus, 1758) & 1 \\
\hline Cephalotes sp.2 & 1 \\
\hline Cephalotes sp.3 & 1 \\
\hline Crematogaster levior Longino, 2003 & 11 \\
\hline Crematogaster aff. stollii & 1 \\
\hline Crematogaster sp.2 & 1 \\
\hline Crematogaster sp.4 & 1 \\
\hline Pheidole biconstricta Mayr, 1870 & 2 \\
\hline Pheidole sp.2 & 1 \\
\hline Procryptocerus sp.1 & 1 \\
\hline Solenopsis sp.1 & 1 \\
\hline Solenopsis sp.2 & 1 \\
\hline
\end{tabular}

\begin{tabular}{l|c}
\hline \multicolumn{2}{l}{ Paraponerinae } \\
\hline \multicolumn{2}{c}{ Paraponera clavata (Fabricius, 1775) } \\
\hline Ponerinae \\
\hline \multicolumn{2}{c}{ Anochetus sp.1 } \\
\hline Total de registros & 1 \\
\hline
\end{tabular}

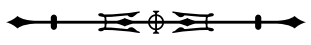




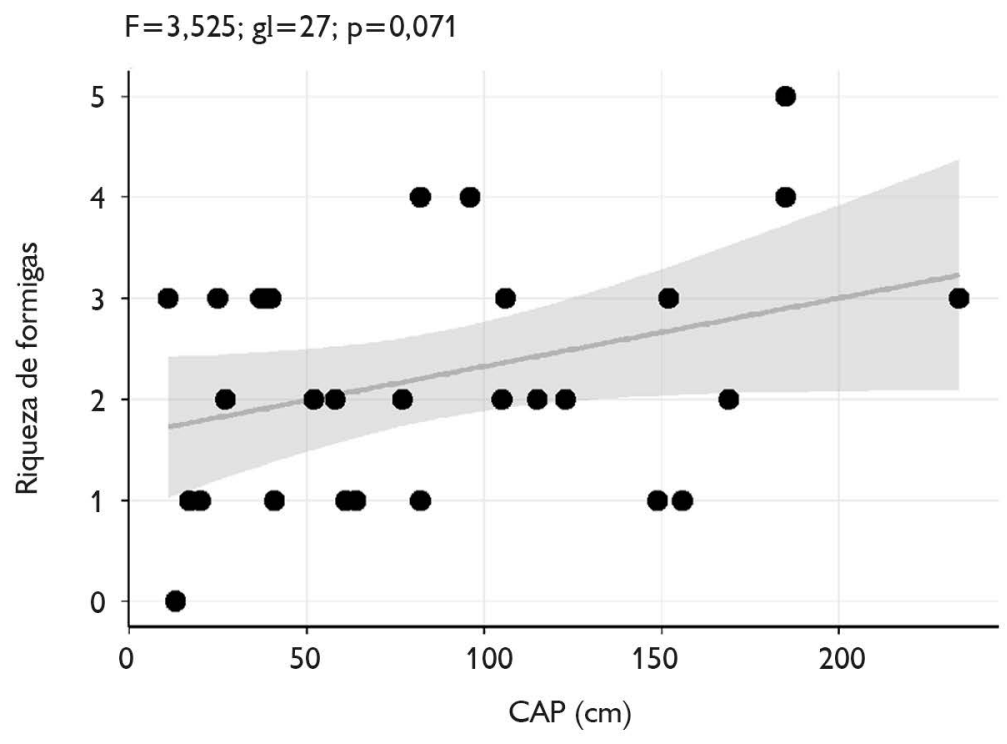

Figura 2. Relação entre a riqueza de espécies de formigas arborícolas e a circunferência à altura do peito (CAP) de árvores na Floresta Nacional de Caxiuanã, Melgaço, Pará, Brasil.

dominantes, das espécies de formigas presentes no estrato arbóreo (Baccaro et al., 2015; Ribeiro et al., 2013).

A conectividade da vegetação é um fator preponderante a influenciar o padrão de comunidades em escala local. Este fator, característico em florestas tropicais preservadas, maximiza, por exemplo, a eficiência de forrageamento de formigas (Jimenez-Soto et al., 2019; Clay et al., 2010), além de contribuir para mudanças na riqueza e na composição de espécies (Adams et al., 2017; Lassau \& Hochuli, 2004). A conectividade facilita o acesso a outras árvores de entorno e, na ausência de espécies agressivas e/ou dominantes, favorece o forrageamento de formigas entre diferentes árvores, permitindo que os recursos sejam igualmente acessados pela assembleia de formigas. Isso explicaria, em parte, a ausência de relação entre a circunferência das árvores e a comunidade de formigas. Por outro lado, árvores sem conectividade podem se comportar como ilhas, impedindo o movimento da fauna entre diferentes árvores e preservando a assembleia local (Adams et al., 2017), contribuindo com a manutenção da riqueza de espécies, independentemente da circunferência arbórea.

A inexistência da relação entre assembleia de formigas e a circunferência das árvores também pode estar vinculada ao comportamento agressivo ou dominante de algumas formigas arbóreas encontradas neste estudo. É possível que a complexidade estrutural do ambiente forneça um habitat adequado para o estabelecimento de grandes colônias de formigas agressivas, comuns em florestas tropicais úmidas (Dejean \& Corbara, 2003). Este estabelecimento impossibilita a chegada ou a nidificação de espécies menos competitivas, diminuindo a riqueza e impedindo a rotatividade das espécies. De fato, entre os gêneros mais frequentes que encontramos, Dolichoderus é dominante em seus habitats (Baccaro et al., 2015), enquanto Camponotus e Crematogaster podem influenciar a distribuição de espécies menos competitivas em florestas tropicais (Dejean et al., 2003).

De toda a fauna de formiga que amostramos, alguns registros merecem destaque. Duas das espécies mais

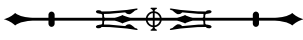




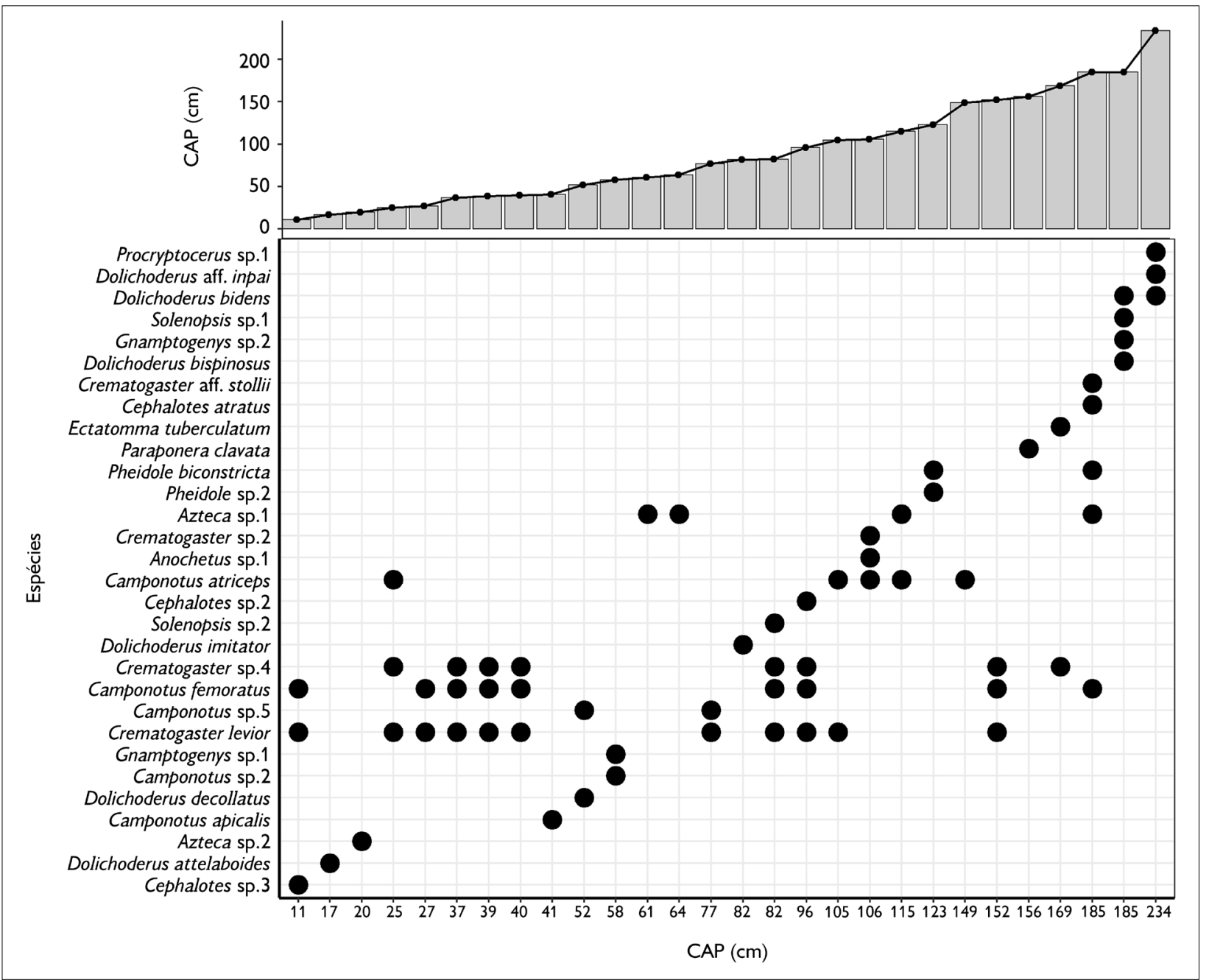

Figura 3. Relação entre a presença de espécies de formigas arborícolas com um gradiente de circunferência à altura do peito (CAP) de árvores na Floresta Nacional de Caxiuanã, Melgaço, Pará, Brasil. As espécies de formigas foram ordenadas de acordo com a média da CAP das árvores em que foram registradas.

frequentes encontradas no presente estudo, Crematogaster levior e Camponotus femoratus, constituem os jardins de formigas mais comumente encontrados na floresta amazônica (Vicente \& Izzo, 2017; Orivel \& Leroy, 2011). Nesta interação, embora não exista preferência por forrageamento arbóreo, há diminuição dessa atividade no solo quando se tem maior conectividade entre árvores (Vicente \& Izzo, 2017), o que pode explicar a alta ocorrência de ambas as espécies encontrada no presente estudo. Gêneros como Gnamptogenys Roger, 1863 e Anochetus Mayr, 1861 não são frequentemente associados à vegetação. Apesar disso, registros dessa natureza não são incomuns (ver Pringle et al., 2019; Ribas et al., 2003) e a literatura aponta a existência de espécies arborícolas ou com forrageamento neste substrato para ambos os gêneros (ver Baccaro et al., 2015).

Durante as coletas, nós observamos que espécies dominantes e/ou agressivas, como Azteca sp. 1, Dolichoderus bispinosus (Olivier, 1792) e Dolichoderus decollatus Smith, 1858, localizaram e monopolizaram o recurso. Estas espécies se posicionaram por toda a borda da armadilha,

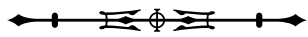


dominando o recurso e impedindo o acesso de outras espécies (Apêndice). No entanto, essa detecção não foi simultânea à colocação da armadilha, o que permitiu o acesso de espécies menos competitivas à fonte de recurso. Por outro lado, a armadilha que capturou Dolichoderus attelaboides (Fabricius, 1775) não coletou outras espécies de formigas, possivelmente devido à presença de um ninho desta espécie na árvore amostrada. Isso facilitou a detecção do recurso e o domínio imediato da armadilha, evitando o acesso de espécies menos competitivas.

\section{CONCLUSÃO}

A disponibilidade de recursos é um fator modulador das comunidades, por isso esperávamos que, para formigas arborícolas, esse fator se refletisse em árvores de maiores CAP, uma vez que forneceria maiores áreas para forragemento e locais de nidificação. Contudo, nossos resultados demonstram que a circunferência das árvores não explica variações na riqueza e na composição dessas formigas. Fatores mais diretamente associados à heterogeneidade ambiental, como conectividade das árvores e espécies dominantes, devem ser considerados em estudos futuros, a fim de proporcionar conhecimento quanto aos impulsionadores de diversidade animal no estrato vertical das florestas tropicais em escala local.

\section{AGRADECIMENTOS}

O presente estudo foi realizado com apoio da Coordenação de Aperfeiçoamento de Pessoal de Nível Superior - Brasil (CAPES) - código de financiamento 001, a quem os autores agradecem pelas bolsas recebidas. Aos professores do Programa de Pós-Graduação em Zoologia da Universidade Federal do Pará, Gleomar Maschio, Marcos Pérsio e Maria Cristina Costa, pela dedicação, sensibilidade, empenho e oportunidades disponibilizados aos autores durante a realização da disciplina "Ecologia de campo da floresta amazônica", de onde os resultados deste estudo são provenientes. Aos dois revisores anônimos, pelos valiosos comentários e sugestões a este estudo.

\section{REFERÊNCIAS}

ADAMS, B. J., S. A. SCHNITZER \& S. P. YANOVIAK, 2017. Trees as islands: canopy ant species richness increases with the size of lianafree trees in a Neotropical forest. Ecography 40(9): 1067-1075. DOI: https://doi.org/10.1111/ecog.02608.

AUGUIE, B., 2017. gridExtra: miscellaneous functions for "grid" graphics. R package version 2.3. Disponível em: https://CRAN.Rproject.org/package = gridExtra. Acesso em: 25 janeiro 2020.

BACCARO, F. B., R. M. FEITOSA, F. FERNANDEZ, I. O. FERNANDES, T. J. IZZO, J. L. P. SOUSA \& R. SOLAR, 2015. Guia para os gêneros de formigas do Brasil: 1-388. Editora INPA, Manaus. DOI: https://doi.org/10.5281/zenodo.32912.

BESTELMEYER, B. T., D. AGOSTI, L. E. ALONSO, C. R. F. BRANDÃO, W. L. BROWNJR., J. H. C. DELABIE \& R. SILVESTRE, 2000. Field techniques for the study of ground-living ants: an overview, description, and evaluation. In: D. AGOSTI, J. D. MAJER, L. E. ALONSO \& T. R. SCHULTZ (Ed.): Ants: standard methods for measuring and monitoring biodiversity: 122-144. Smithsonian Institution, Washington.

CAMPOS, R. I., C. T. LOPES, W. C. S. MAGALHÃES \& H. L. VASCONCELOS, 2008. Estratificação vertical de formigas em cerrado strictu sensu no Parque Estadual da Serra de Caldas Novas, Goiás, Brasil. Iheringia, Série Zoologia 98(3): 311-316. DOI: https:// doi.org/10.1590/S0073-47212008000300004.

CLARKE, K. R., 1993. Non-parametric multivariate analysis of changes in community structure. Australian Journal of Ecology 18(1): 117-143. DOI: https://doi.org/10.1111/1.1442-9993.1993.tb00438.x.

CLAY, N. A., M. BAUER, M. SOLIS \& S. P. YANOVIAK, 2010. Arboreal substrates influence foraging in tropical ants. Ecological Entomology 35(4): 417-423. DOI: https://doi.org/10.1111/j.13652311.2010.01197.x.

CRAWLEY, M. J., 2013. The R book. Wiley, Chichester, West Sussex, United Kingdom.

DEJEAN, A. \& B. CORBARA, 2003. A review of mosaics of dominant ants in rainforests and plantations. In: Y. BASSET, $V$. NOVOTNY, S. E. MILLER \& R. L. KITCHING (Ed.): Arthropods of tropical forests: spatio-temporal dynamics and resource use in the canopy: 341-347. Cambrige University Press, Cambrige.

DEJEAN, A., B. CORBARA, F. FERNÁNDEZ \& J. H. DELABIE, 2003. Mosaicos de hormigas arbóreas en bosques y plantaciones tropicales. In: F. FERNÁNDEZ (Ed.): Introducción a las hormigas de la región Neotropical: 149-158. Instituto de Investigación de Recursos Biológicos Alexander von Humboldt, Bogotá.

FAGUNDES, R., D. V. ANJOS, R. CARVALHO \& K. DELCLARO, 2015. Availability of food and nesting-sites as regulatory mechanisms for the recovery of ant diversity after fire disturbance. Sociobiology 62(1): 1-9. DOI: https://doi.org/10.13102/ sociobiology.v62i1.1-9.

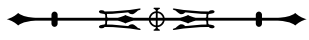


HASHIMOTO, Y., Y. MORIMOTO, E. S. WIDODO, M. MOHAMED \&J. R. FELLOWES, 2010. Vertical habitat use and foraging activities of arboreal and ground ants (Hymenoptera: Formicidae) in a bornean tropical rainforest. Sociobiology 56: 435-448.

JIMENEZ-SOTO, E., J. R. MORRIS, D. K. LETOURNEAU \& S. M. PHILPOTT, 2019. Vegetation connectivity increases ant activity and potential for ant-provided biocontrol services in a tropical agroforest. Biotropica 51(1): 50-61. DOI: https://doi.org/10.1111/ btp.12616.

KLIMES, P., C. IDIGEL, M. RIMANDAI, T. M. FAYLE, M. JANDA, G. D. WEIBLEN \& V. NOVOTNY, 2012. Why are there more arboreal ant species in primary than in secondary tropical forests? Journal of Animal Ecology 81(5): 1103-1112. DOI: https://doi. org/10.1111/j.1365-2656.2012.02002.x.

LASSAU, S. A. \& D. F. HOCHULI, 2004. Effects of habitat complexity on ant assemblages. Ecography 27: 157-164.

LISBOA, P. L. B., 1997. Caxiuanã: 1-446. Museu Paraense Emílio Goeldi, Belém.

OKSANEN, J., F. G. BLANCHET, M. FRIENDLY, R. KINDT, P. LEGENDRE, D. MCGLINN, P. R. MINCHIN, R. B. O'HARA, G. L. SIMPSON, P. SOLYMOS, M. HENRY, H. STEVENS, E. SZOECS \& H. WAGNER, 2019. vegan: community ecology package. $R$ package version 2.5-6. Disponível em: https://CRAN.R-project. org/package= vegan. Acesso em: 25 janeiro 2020.

ORIVEL, J. \& C. LEROY, 2011. The diversity and ecology of ant gardens (Hymenoptera: Formicidae, Spermatophyta: Angiospermae). Myrmecological News 14: 73-85

POWELL, S., A. N. COSTA, C. T. LOPES \& H. L. VASCONCELOS, 2011. Canopy connectivity and the availability of diverse nesting resources affect species coexistence in arboreal ants. Journal of Animal Ecology 80(2): 352-360. DOI: https://doi.org/10.1111/ j.1365-2656.2010.01779.x.

PRINGLE, E. G., T. F. SANTOS, M. S. GONÇALVES, J. E. HAWES, C. A. PERES \& F. B. BACCARO, 2019. Arboreal ant abundance tracks primary productivity in an Amazonian whitewater river system. Ecosphere 10(10): e02902. DOI: https://doi.org/10.1002/ ecs2.2902.

R CORE TEAM, 2019. R: a language and environment for statistical computing. R Foundation for Statistical Computing, Vienna, Austria. Disponível em: https://www.R-project.org/. Acesso em: 25 janeiro 2020.

RIBAS, C. R., J. H. SCHOEREDER, M. PIC \& S. M. SOARES, 2003. Tree heterogeneity, resource availability, and larger scale processes regulating arboreal ant species richness. Australian Journal of Ecology 28(3): 305-314. DOI: https://doi.org/10.1046/j.14429993.2003.01290.x.
RIBEIRO, S. P., N. B. ESPÍRITO-SANTO, J. H. C. DELABIE \& J. D. MAJER, 2013. Competition, resources and the ant (Hymenoptera: Formicidae) mosaic: a comparison of upper and lower canopy. Myrmecological News 18: 113-120.

RICKLEFS, R. E. \& D. SCHLUTER, 1993. Species diversity in ecological communities: historical and geographical perspectives. University of Chicago Press, Chicago.

SILVA, A. S. L., S. S. ALMEIDA \& C. S. ROSÁRIO, 2003. Flórula fanerogâmica da Estação Científica Ferreira Penna (ECFPn): caracterização dos ecossistemas e lista preliminar de espécies.

Resumos do Seminário de 10 anos de atividades da ECFPn, Caxiuanã Seção 1

SOUSA-SOUTO, L., P. M. G. FIGUEIREDO, B. G. AMBROGI, A. C. F. OLIVEIRA, G. T. RIBEIRO \& F. S. NEVES, 2016. Composition and richness of arboreal ants in fragments of Brazilian Caatinga: effects of secondary succession. Sociobiology 63(2): 762-769. DOI: http:// dx.doi.org/10.13102/sociobiology.v63i2.909.

STEIN, A., K. GERSTNER \& H. KREFT, 2014. Environmental heterogeneity as a universal driver of species richness across taxa, biomes and spatial scales. Ecology Letters 17(7): 866-880. DOI: http://dx.doi.org/10.1111/ele.12277.

TANAKA, H. O., S. YAMANE \& T. ITIOKA, 2010. Within-tree distribution of nest sites and foraging areas of ants on canopy trees in a tropical rainforest in Borneo. Population Ecology 52(1): 147-157. DOI: https://doi.org/10.1007/s10144-009-0172-2.

VASCONCELOS, H. L., J. B. MARAVALHAS, R. M. FEITOSA, R. PACHECO, K. C. NEVES \& A. N. ANDERSEN, 2017. Neotropical savanna ants show a reversed latitudinal gradient of species richness, with climatic drivers reflecting the forest origin of the fauna. Journal of Biogeography 45(1): 248-258. DOI: https://doi.org/10.1111/jbi.13113.

VICENTE, R. E. \& T. J. IZZO, 2017. Defining habitat use by the parabiotic ants Camponotus femoratus (Fabricius, 1804) and Crematogaster levior Longino, 2003. Sociobiology 64(4): 373-380. DOI: http://dx.doi.org/10.13102/sociobiology.v64i4.1228.

WICKHAM, H., 2016. ggplot2: elegant graphics for data analysis. Springer-Verlag, New York.

YAMAZAKI, L., J. DAMBROZ, E. MEURER, V. F. VINDICA, J. H. C. DELABIE, M. I. MARQUES \& L. D. BATTIROLA, 2016. Ant community (Hymenoptera: Formicidae) associated with Callisthene fasciculata (Spr.) Mart. (Vochysiaceae) canopies in the Pantanal of Poconé, Mato Grosso, Brazil. Sociobiology 63(2): 735-743. DOI: http://dx.doi.org/10.13102/sociobiology.v63i2.824.

ZUUR, A. F., E. N. IENO \& C. S. ELPHICK, 2010. A protocol for data exploration to avoid common statistical problems. Methods in Ecology and Evolution 1(1): 3-14. DOI: https://doi.org/10.1111/ j.2041-210X.2009.00001.x. 
Apêndice. Espécies dominantes Dolichoderus decollatus Smith, 1858 (A) e Dolichoderus bispinosus (Olivier, 1792) (B) monopolizando as armadilhas pitfall na vegetação. Fotos: Rony Peterson Santos Almeida (2017).

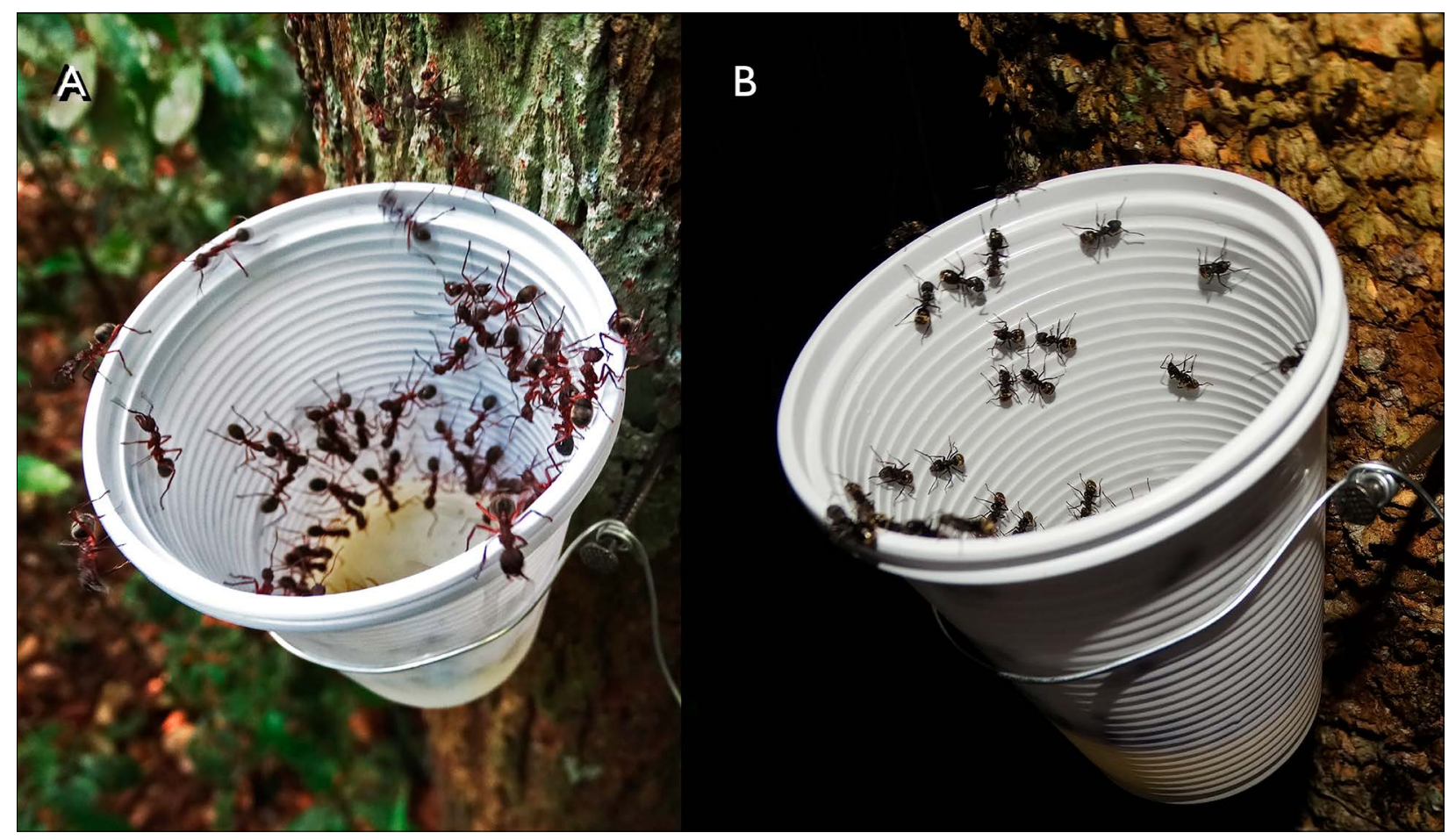


